\title{
Perception of Human Activities A Means to Support Connectedness Between the Elderly and Their Caregivers
}

\author{
Kadian Davis ${ }^{1}$, Evans Owusu ${ }^{2}$, Carlo Regazzoni ${ }^{3}$, Lucio Marcenaro ${ }^{3}$, Loe Feijs ${ }^{1}$ and Jun $\mathrm{Hu}^{1}$ \\ ${ }^{1}$ Department of Industrial Design, Eindhoven University of Technology, Den Dolech 2, 5612 AZ, Eindhoven, \\ The Netherlands \\ ${ }^{2}$ Independent Researcher, Eindhoven, The Netherlands \\ ${ }^{3}$ Electrical, Electronics and Telecommunication Engineering and Naval Architecture Department, University of Genoa, \\ Via Opera Pia 11a, 16145, Genova, Italy \\ \{K.A.Davis, J.Hu, L.M.G.Feijs\}@tue.nl,Owboateng@gmail.com,lucio.marcenaro@unige.it, carlo@dibe.unige.it
}

Keywords: $\quad$ Activity Recognition, Monitoring, Sensors, Smart-phone, Perception, Connectedness.

Abstract: This position paper describes a smart-phone based activity recognition system for improving social connectedness between caregivers and their elderly relatives. Sensing technologies can enable real-time monitoring to provide activity recognition in order to support health and safety among the elderly who are living independently. However, most existing activity recognition systems are focused on using sensors for unidirectional monitoring of emergency cases in particular, fall detection. Motivated by the desire to utilize bidirectional activity recognition to improve connectedness between an ageing population and their caregivers, we describe our planned approach to investigate how the perception of a caregiver's activities by a senior citizen and vice versa can aid in improving social connectedness. To investigate this perception, activity states will be transformed into an information visualization into the caregiver's home and vice versa without overt communication from participants. Findings are expected to provide further insight on the extent to which perception of human activities increase social connectedness.

\section{INTRODUCTION}

With the growth of demographic ageing within the European Union, formal and informal care services are increasingly becoming concerned about the fiscal burden and demands of the ageing population. Consequently, these institutions are in favour of home care solutions. As discussed in Davis et al. (2015), there are inadequate resources such as nurses, which induce cost; and consequently independent living is forced. In contrast, despite wanting to live 'separate' lives from their elderly counterparts, caregivers often experience worry or uncertainty about their elderly relative's health and well-being.

For many years, a number of scholars have associated social isolation and loneliness with old age (Sheldon et al., 1948; Halmos, 2013). In general, retirement, mobility-impairment, increased isolation, death of loved ones and kin-separation due to globalization may cause loneliness in ageing societies. Furthermore, research has found a number of health risks associated with loneliness and social isolation including high mortality risks, cardiovascular and infectious diseases, cognitive deterioration and depres- sion (Becker et al., 1998; Stafford et al., 2011; Steptoe et al., 2013). However, epidemiological research suggests that strong social ties play a critical role in enhancing the elderly's psychological and physiological well-being (Umberson and Montez, 2010).

Social participation has been incorporated into the research and public policies of ageing societies as shown in (Landabaso and Letter, 2013). As a result, this enables social cohesion through active involvement in volunteering services, clubs and associations. In turn, this increases a sense of belongingness and also influences quality of life, which is critical for the sustainable development of ageing societies.

Human activity recognition (HAR) systems can help in reducing the challenges of supporting elderly independent living and reducing the burden of their caregivers. Moreover, HAR systems provide a plethora of opportunities for automatic recognition of activities of daily living (ALDs) in the context of health and elderly care such as fall detection (Bourke et al., 2007), classifying activities of Parkinson's disease patients (Rodriguez-Martin et al., 2013) and encouraging physical activity (Consolvo et al., 2008). However, most of the existing applications are fo- 
cused on detecting potentially dangerous situations and generating automatic alarms in the case of an emergency (Rashidi and Mihailidis, 2013).

\subsection{Research Goals}

Social Connectedness can be described as a sense of belongingness based on the appraisal of having enough close contacts (Van Bel et al., 2008). In this position paper, we hope to improve social connectivity through the perception of activity states generated from smart-phone sensors. This work forms part of the proposed experiment of Davis et al. (2015), which aims to induce social presence through subtle awareness of activity and emotional states between the caregivers and their elderly relatives.

The ubiquitous cell-phone provides a rich platform for human activity recognition as shown in (Anguita et al., 2012). The inclusion of sensors namely the camera, accelerometer and gyroscope, its unobtrusiveness and communication features (WIFI, 3G and Bluetooth) add to the smart-phone's appeal for enabling human activity recognition (Ustev et al., 2013). For the purposes of this research, we hope to apply the notion of abstraction (i.e. highlighting key information contained in the data and suppressing irrelevant details as discussed in (Cavaco, 2014) to investigate how the elderly and their caregivers will perceive raw activity data versus classified data.

\section{RELATED WORK}

In perceiving human activity, we rely on several information sources from various modalities including sensory, motor and affective processes (Blake and Shiffrar, 2007). Specifically, the human visual system seemingly plays a significant role in understanding human actions and intentions. Furthermore, it is extremely sensitive to human movement and as such it is able to extract socially relevant details (Troje, 2002). This implies that human motion could provide reliable information for discerning affect (Blake and Shiffrar, 2007). For instance, Kaye et al. (2005) confirmed that virtual intimate objects gave a sense of peripheral presence and activity awareness; thus enhancing intimacy in long distance relationships. Therefore, we believe that the perception of human activities can improve social connectedness. For instance, imagine you have an information display showing your distant elderly parent's activities in the periphery of your living room, which is placed in your living room and you suddenly discern that something is wrong and you are prompted to call your mother. Could you recognize specific patterns in the daily activities of your mother? Could you recognize that something was wrong based on the dynamics of the visualization in the absence of direct communication? Would this improve social connectedness between yourself and your elderly parent? We believe that this is possible and in this section we will explore a number of studies focused on awareness systems for elderly care.

\subsection{Awareness Systems Supporting Elderly Care}

Naturally, awareness of others and their activities can directly influence social connectedness by addressing social and emotional needs and possibly initiate communication through other media (Markopoulos and Mackay, 2009; Romero et al., 2007). Early conceptions of awareness systems to support ageing in place were introduced through the Digital Family Portrait (Mynatt et al., 2001) and the CareNetDisplay (Consolvo et al., 2004). The Digital Family Portrait used sensors to collect information (eg., weather and activities) to display a qualitative sense of a senior citizen's daily activities to their families over a mediated environment. The CareNetDisplay was an extension of the previous ideas of the Digital Family Portrait and consequently; the researchers amplified an older person's photograph with details about their daily life including activities, moods, medication, falls, meals and outings (Consolvo et al., 2004). Later, the Daily Activities Diarist sought to address the weaknesses of the previous awareness systems through its narrative presentation of awareness information (Metaxas et al., 2007). These earlier systems demonstrated great potential for facilitating awareness for distant family members who were worried about their elderly relatives or members of an elderly care network offering day-to-day care. However, like the Digital Family Portrait and CareNetDisplay, the Diarist reflected an unbalanced communication channel and in most cases portrayed unidirectional monitoring where the caregiver observed their elderly relatives for notification of alarming situations. Unidirectional monitoring involves the deployment of wearable devices and other intrusive sensors all around a senior's home. This is invasive and consequently violates the privacy and dignity rights of the elderly population.

More recent communication mediated systems that support peripheral awareness for connecting remote families include the following: the ASTRA awareness system for connecting households and mobile family members Romero et al. (2007) and SoPresent - an awareness system for connecting remote 
households Dadlani et al. (2014). In sum, both Astra and SoPresent attempted to address the asymmetrical communication concerns of the previously discussed research by sharing moments and experiences. However, they were designed to support connectivity between families of remote households, which though related is not the specific context of this research. In our research, we are interested in understanding how the elderly and their caregivers will perceive the activity data presented in the periphery and how this acquired knowledge improves social connectivity.

\section{PROPOSED ACTIVITY RECOGNITION PLAN}

We have chosen an Android based platform as a means for activity recognition because of its embedded sensors such as the accelerometer and gyroscope. In addition, Android's openness provides existing resources (source modifications and open tools), which facilitate the inexpensive development of context-awareness tools. Therefore, we have developed the Accelerometer Gyrometer Logger (available on Google Play) to collect accelerometric and gyroscopic sensor readings at a frequency of $50 \mathrm{~Hz}$. Specific properties will be extracted (eg. mean, standard deviation, entropy etc.) from this data to detect the following activities: walking, standing, laying, sitting, walking upstairs and downstairs, running and cycling for both the elderly and their caregivers.

Several studies have shown that the Support Vector machine (SVM) is the most popular classification method in comparison to the quadratic classifier, knearest neighbor algorithm and artificial neural networks. For instance, Ravi et al. (2005) demonstrated that SVM gives one of the best accuracies for recognizing human activities achieving over $99.4 \%$ accuracy for boosted SVM. Moreover, Anguita et al. (2012) adapted the standard SVM to exploit fixedpoint arithmetic to reduce computational complexity. This approach enabled them to deploy the smartphone inbuilt accelerometer and gyrometer sensors while maintaining normal battery lifespans for other shared resources on the phone. The adapted multiclass SVM obtained similar accuracy compared with the traditional SVM. In this position paper, we propose to adopt the multi-class SVM algorithm inspired by Anguita et al. (2012) to train and classify our data for recognizing the activities we have mentioned earlier.

\subsection{Data Collection}

Anguita et al. (2012) have made publicly available, a HAR training dataset (accelerometer and gyrometer) of 30 volunteers in the age range of 19-48 years. In their experiment, each volunteer was instructed to perform the following activities: sitting, standing, walking, lying, walking upstairs and walking downstairs while wearing a smart-phone on their waist. The experiments were video recorded to enable them to label the data. We will adopt a similar approach to collect additional datasets to include activities such as running and cycling. Our dataset together with the existing dataset will be used to train the multi-class SVM classifier for activity recognition. However, our study will include a larger sample space with our participants ranging between 18-90 years, who are living mostly in the Netherlands. Two sets of training data will be used, one for participants aged 18-59 years and the other for participants aged 60-90 years. We assume the elderly population will exhibit a slower speed of motion in comparison to their caregivers. The autographer wearable camera will be mounted unto the participant's bodies to capture daily activities for labelling and interpreting the data. Also, we will utilize the caregivers and their elderly relatives own homes as test-beds for our data collection. For the purposes of this experiment, we assume that the homes of the caregivers and their elderly relatives are furnished with staircases, which is typical of Dutch family homes. The selected activities include typical activities of daily living, which are commonly found in validated scales such as (Reisberg et al., 2001). Also, we used the following articles (Chavarriaga et al., 2013; Cook et al., 2009) as references for compiling our scenario.

\subsubsection{Scenario Script}

For our experiment, we will ask each participant to perform the following activities in sequence.

- Telephone Use (Sitting): The subject will dial a specific number listed on an instruction sheet and write down the instructions given on the recorded message. The stationary and cellular phone will be located on the dining room table.

- Cleaning (Walking): Participants will be asked to vacuum the floor of their living rooms.

- Exercising (Running and Cycling): Outside of their own homes, able bodied participants will be asked to jog on the spot for a few minutes. Moreover, those who are able to cycle will be asked to ride a bicycle for a few minutes. Note well that this scenario is more applicable to the caregivers. 
- Hand Washing (Standing): Participants will be asked to wash their hands in the bathroom face basin using their own toiletries.

- Collect Ingredients (Walking Upstairs and Downstairs): Each Participant will be asked to collect some ingredients, which will be placed on the stair-head.

- Preparing a Snack (Walking and Standing): Participants will be asked to prepare a snack and beverage in accordance with the recorded instructions.

- Resting (Lying): Participants will be asked to lay on the couch in their living rooms.

Moreover, the data sets generated from the above mentioned activities will serve as ground truth for validating the perceptions of both the caregivers and their elderly relatives.

\section{THE EXPERIMENT}

In this paper, we will investigate the extent to which the perception of the activities of the elderly by their caregivers and vice versa help to improve bonding. Moreover, we seek to understand how much hidden information would be perceived from the unclassified accelerometer data and the classified data, which has a direct correlation between the level of activity and the jagged edges of the output graph.

\subsection{Proposed Methodology}

We will conduct a preliminary study applying the within subjects design to a group comprising of ten caregivers and ten elderly participants. To reduce the carry-over effects of this experimental design, we will divide the group into two groups A and B. This implies, that we will show Group A the classified activity display and subsequently show the unclassified activity display. For Group B, we will first show the unclassified activity display and then the classified activity display. Figures 1 and 2 summarize the experimental approaches.

In the classification approach, we will extract and select these features (standard deviation, mean, entropy etc), which will be used to train the multi-class SVM classifier. Signals from the both the elderly and the caregivers will be streamed to a server, which will perform feature extraction and selection, and subsequently classify the activities. The activities will be grouped into low, medium and high levels according to table one. These levels of activities will be relayed to android tablets, positioned like a photo-frame in

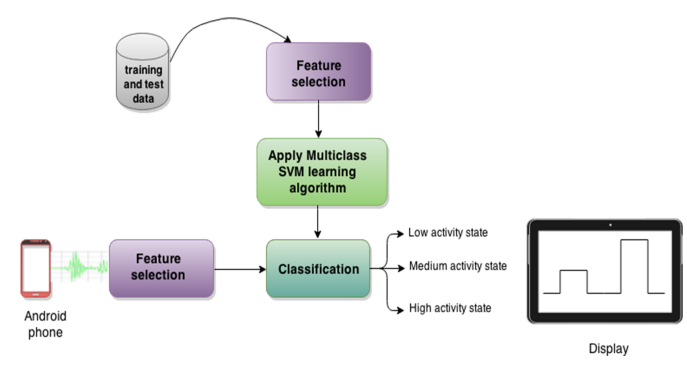

Figure 1: Set-up I showing the classification approach.

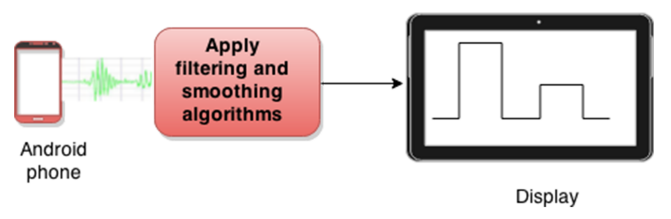

Figure 2: Set-up II showing the unclassified approach.

Table 1: Table showing activity classification levels.

\begin{tabular}{|c|c|}
\hline Activities & Activity Levels \\
\hline lying & low \\
\hline sitting & low \\
\hline standing & medium \\
\hline walking & medium \\
\hline walking downstairs & medium \\
\hline running & high \\
\hline cycling & high \\
\hline walking upstairs & high \\
\hline
\end{tabular}

the homes of both the caregivers and their elderly relatives and will be rendered using simple line graphs (Figure 3).

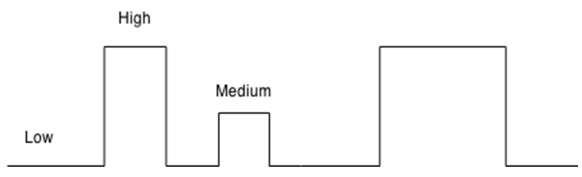

Figure 3: Line graph showing the activity levels.

The dual display of the activities of both the caregiver and their elderly relatives shows a bidirectional communication channel, which we believe is a necessary component for reducing the 'Big Brother' role of caregivers. As shown in set-up II, the experiment is repeated but this time, the original raw accelerometer data is filtered, smoothened and approximated to a similar line graph as displayed in the set-up I.

Our choice of simple line graphs to display the activities is not random. Line graphs are one of the most common means of visualizing time-series data. 
They are able to easily display data, facilitate comparison and reveal trends within the data. As shown in Figure 3, we believe it will be easier to associate low, medium and high slopes with low, medium and high activity levels respectively. Therefore, this visual rendering will portray the same aesthetic quality and visual complexity for both displays.

\subsection{Evaluation}

To determine the usefulness of the proposed approach we hope to investigate the following:

- How does the experimental subjects perceive both displays and their preference?

- Did the elderly participants recognize certain patterns in the activities of their caregivers and vice versa?

- When they observe the display, what are their perceptions of their caregiver's activities?

- Will the application trigger social behaviour? For instance, will we see an increase in phone calls?

Furthermore, to investigate social connectedness we will utilize the validated scales such as the Inclusion of Other in the Self scale (IOS) as discussed in (Aron et al., 1992) and the IPO Social Presence questionnaire detailed in (De Greef and Ijsselsteijn, 2001).

\section{CONCLUSIONS AND FUTURE WORK}

We have presented our proposed approach for perception of activities from smart-phone generated data to increase bonding relations between the elderly and their caregivers. Furthermore, we have described two techniques for investigating the participants' perceptions of the activity displays and how their perceptions would support social connectivity between the generations. Firstly, we proposed the popular SVM technique for the activity classification display and secondly we proposed an abstraction of the original raw accelerometric and gyroscopic data for the unclassified display. Provided that the results of this experiment are favourable, we have a variety of activity displays in mind and we hope to implement them in the near future. Our major long term goal is to improve bonding and care by designing a system in which the elderly and their caregivers can interact through other signals (mainly physiological signals), among them unconscious signals. In subsequent experiments, we will implement a covert lighting application, which will provide contextual awareness of activity and emotional states of both the caregivers and their elderly counterparts. Therefore, we believe this subtle contextual awareness will facilitate the understanding of the affective and activity states of both parties and in turn will help them to respond with the appropriate behaviour. Consequently, this could contribute to improving social connectedness.

\section{ACKNOWLEDGEMENTS}

This work was supported in part by the Erasmus Mundus Joint Doctorate (EMJD) in Interactive and Cognitive Environments (ICE), which is funded by Erasmus Mundus under the FPA no. 2010-2012.

\section{REFERENCES}

Anguita, D., Ghio, A., Oneto, L., Parra, X., and ReyesOrtiz, J. L. (2012). Human activity recognition on smartphones using a multiclass hardware-friendly support vector machine. In Ambient assisted living and home care, pages 216-223. Springer.

Aron, A., Aron, E. N., and Smollan, D. (1992). Inclusion of other in the self scale and the structure of interpersonal closeness. Journal of personality and social psychology, 63(4):596.

Becker, T., Leese, M., Clarkson, P., Taylor, R., Turner, D., Kleckham, J., and Thornicroft, G. (1998). Links between social networks and quality of life: an epidemiologically representative study of psychotic patients in south london. Social Psychiatry and Psychiatric Epidemiology, 33(7):299-304.

Blake, R. and Shiffrar, M. (2007). Perception of human motion. Annu. Rev. Psychol., 58:47-73.

Bourke, A., O'brien, J., and Lyons, G. (2007). Evaluation of a threshold-based tri-axial accelerometer fall detection algorithm. Gait \& posture, 26(2):194-199.

Cavaco, C. A. d. Q. S. (2014). New visualization model for large scale biosignals analysis.

Chavarriaga, R., Sagha, H., Calatroni, A., Digumarti, S. T., Tröster, G., Millán, J. d. R., and Roggen, D. (2013). The opportunity challenge: A benchmark database for on-body sensor-based activity recognition. Pattern Recognition Letters, 34(15):2033-2042.

Consolvo, S., McDonald, D. W., Toscos, T., Chen, M. Y., Froehlich, J., Harrison, B., Klasnja, P., LaMarca, A., LeGrand, L., Libby, R., et al. (2008). Activity sensing in the wild: a field trial of ubifit garden. In Proceedings of the SIGCHI Conference on Human Factors in Computing Systems, pages 1797-1806. ACM.

Consolvo, S., Roessler, P., and Shelton, B. E. (2004). The carenet display: lessons learned from an in home evaluation of an ambient display. In UbiComp 2004: Ubiquitous Computing, pages 1-17. Springer. 
Cook, D., Schmitter-Edgecombe, M., Crandall, A., Sanders, C., and Thomas, B. (2009). Collecting and disseminating smart home sensor data in the casas project. In Proceedings of the CHI Workshop on Developing Shared Home Behavior Datasets to Advance HCI and Ubiquitous Computing Research.

Dadlani, P., Gritti, T., Shan, C., de Ruyter, B., and Markopoulos, P. (2014). Sopresent: An awareness system for connecting remote households. Ambient Intelligence, page 67.

Davis, K., Hu, J., Feijs, L., and Owusu, E. B. (2015). Social hue: A subtle awareness system for connecting the elderly and their caregivers. In Pervasive Computing and Communications Workshops (PERCOM Workshops), 2015 IEEE International Conference on. IEEE. In press.

De Greef, P. and Ijsselsteijn, W. A. (2001). Social presence in a home tele-application. CyberPsychology \& Behavior, 4(2):307-315.

Halmos, P. (2013). Solitude and privacy: A study of social isolation, its causes and therapy. Routledge.

Kaye, J., Levitt, M. K., Nevins, J., Golden, J., and Schmidt, V. (2005). Communicating intimacy one bit at a time. In CHI'05 extended abstracts on Human factors in computing systems, pages 1529-1532. ACM.

Landabaso, M. and Letter, L. D. (2013). Guide to social innovation. page 71.

Markopoulos, P. and Mackay, W. (2009). Awareness systems: Advances in theory, methodology and design. Springer Science \& Business Media.

Metaxas, G., Metin, B., Schneider, J., Markopoulos, P., and De Ruyter, B. (2007). Daily activities diarist: supporting aging in place with semantically enriched narratives. In Human-Computer Interaction-INTERACT 2007, pages 390-403. Springer.

Mynatt, E. D., Rowan, J., Craighill, S., and Jacobs, A. (2001). Digital family portraits: supporting peace of mind for extended family members. In Proceedings of the SIGCHI conference on Human factors in computing systems, pages 333-340. ACM.

Rashidi, P. and Mihailidis, A. (2013). A survey on ambientassisted living tools for older adults. IEEE journal of biomedical and health informatics, 17(3):579-590.

Ravi, N., Dandekar, N., Mysore, P., and Littman, M. L. (2005). Activity recognition from accelerometer data. In $A A A I$, volume 5, pages 1541-1546.

Reisberg, B., Finkel, S., Overall, J., Schmidt-Gollas, N., Kanowski, S., Lehfeld, H., Hulla, F., Sclan, S. G., Wilms, H.-U., Heininger, K., et al. (2001). The alzheimer's disease activities of daily living international scale (adl-is). International Psychogeriatrics, 13(02):163-181.

Rodriguez-Martin, D., Sama, A., Perez-Lopez, C., Catala, A., Cabestany, J., and Rodriguez-Molinero, A. (2013). Svm-based posture identification with a single waistlocated triaxial accelerometer. Expert Systems with Applications, 40(18):7203-7211.

Romero, N., Markopoulos, P., Van Baren, J., De Ruyter, B., Ijsselsteijn, W., and Farshchian, B. (2007). Connect- ing the family with awareness systems. Personal and Ubiquitous Computing, 11(4):299-312.

Sheldon, J. H. et al. (1948). The social medicine of old age report of an enquiry in wolverhampton. The Social Medicine of Old Age. Report of an Enquiry in Wolverhampton.

Stafford, M., McMunn, A., Zaninotto, P., and Nazroo, J. (2011). Positive and negative exchanges in social relationships as predictors of depression: evidence from the english longitudinal study of aging. Journal of $A g-$ ing and Health.

Steptoe, A., Shankar, A., Demakakos, P., and Wardle, J. (2013). Social isolation, loneliness, and all-cause mortality in older men and women. Proceedings of the National Academy of Sciences, 110(15):5797-5801.

Troje, N. F. (2002). Decomposing biological motion: A framework for analysis and synthesis of human gait patterns. Journal of vision, 2(5):2.

Umberson, D. and Montez, J. K. (2010). Social relationships and health a flashpoint for health policy. Journal of health and social behavior, 51(1 suppl):S54-S66.

Ustev, Y. E., Durmaz Incel, O., and Ersoy, C. (2013). User, device and orientation independent human activity recognition on mobile phones: challenges and a proposal. In Proceedings of the 2013 ACM conference on Pervasive and ubiquitous computing adjunct publication, pages 1427-1436. ACM.

Van Bel, D. T., IJsselsteijn, W. A., and de Kort, Y. A. (2008). Interpersonal connectedness: conceptualization and directions for a measurement instrument. In CHI'08 extended abstracts on Human factors in computing systems, pages 3129-3134. ACM. 


\title{
ICT4AgeingWell 2015
}

International Conference on Information and Communication

Technologies for Ageing Well and e-Health

\section{Proceedings}

\author{
Lisbon, Portugal
}

20 - 22 May, 2015

www.ict4ageingwell.org

SPONSORED BY:

IIISTICC
LOGISTICS PARTNER:

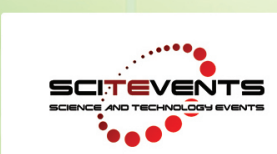

PAPERS AVAILABLE AT:

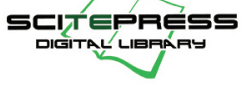




\title{
ICT4AgeingWell 2015
}

\author{
Proceedings of the \\ 1st International Conference on \\ Information and Communication Technologies for \\ Ageing Well and e-Health
}

\author{
Lisbon, Portugal \\ 20 - 22 May, 2015
}

Sponsored by

INSTICC - Institute for Systems and Technologies of Information, Control and Communication

In Cooperation with

ISfTeH - International Society for Telemedicine \& eHealth

EHTEL - European Health Telematics Association

AAL Programme 
Copyright (C) 2015 SCITEPRESS - Science and Technology Publications All rights reserved

Edited by Andreas Holzinger, Carsten Röcker, Ana Fred, Markus Helfert, John O’Donoghue and Martina Ziefle

\section{Printed in Portugal}

ISBN: 978-989-758-102-1

Depósito Legal: 391665/15

http://www.ict4ageingwell.org

ict4ageingwell.secretariat@insticc.org 


\section{BRIEF CONTENTS}

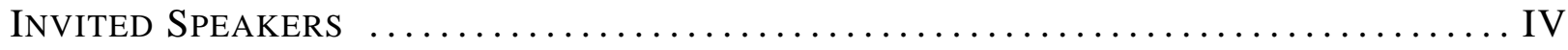

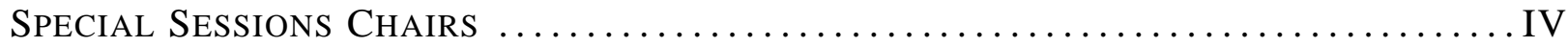

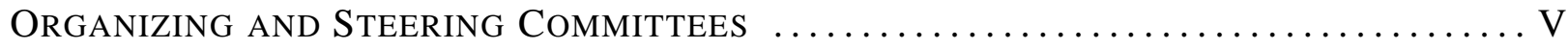

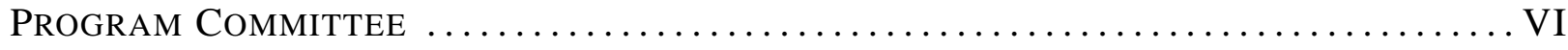

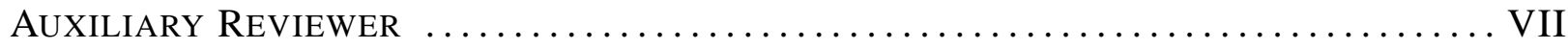

Special Sessions Program Committee $\ldots \ldots \ldots \ldots \ldots \ldots \ldots \ldots \ldots \ldots \ldots \ldots \ldots . . \ldots \ldots$ VIII

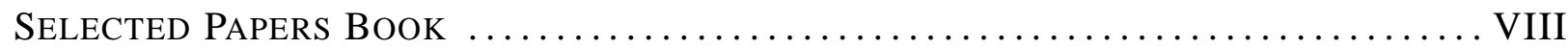

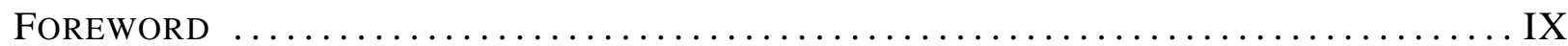

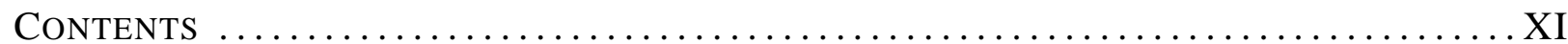




\title{
INVITED SPEAKERS
}

\section{Juan Carlos Augusto}

School of Science and Technology, Middlesex University

U.K.

\author{
Victor Chang \\ Leeds Beckett University
}

U.K.

\section{Thomas Hermann}

CITEC - Center of Excellence Cognitive Interaction Technology, Bielefeld University

Germany

\section{William Molloy}

Centre for Gerontology and Rehabilitation, School of Medicine, UCC

Ireland

\section{SPECial SeSSions Chairs}

\section{Special Session on How Can Digital games Help Older Adults?}

David Kaufman, Simon Fraser University, Canada

\section{Special Session on Social Motivations and Social Benefits of ICT}

Paula Trigueiros, University of Minho, Portugal 


\title{
Organizing AND Steering Committees
}

\section{CONFERENCE CO-CHAIRS}

Markus Helfert, Dublin City University, Ireland

Andreas Holzinger, Medical University Graz, Austria

\section{Program Co-Chairs}

Martina Ziefle, RWTH-Aachen University, Germany

Ana Fred, Instituto de Telecomunicações / IST, Portugal

John O’Donoghue, Imperial College London, U.K.

Carsten Röcker, Ostfestfalen-Lippe UAS and Fraunhofer IOSB-INA, Germany

\section{Proceedings Production}

Susana Andrade, INSTICC, Portugal

Bruno Encarnação, INSTICC, Portugal

Lúcia Gomes, INSTICC, Portugal

Raquel Pedrosa, INSTICC, Portugal

Vitor Pedrosa, INSTICC, Portugal

Sara Santiago, INSTICC, Portugal

José Varela, INSTICC, Portugal

\section{CD-ROM PRODUCTION}

Pedro Varela, INSTICC, Portugal

\author{
Graphics Production and Webdesigner \\ André Lista, INSTICC, Portugal \\ Mara Silva, INSTICC, Portugal
}

SeCretariat

Andreia Pereira, INSTICC, Portugal

WEBMASTER

Susana Ribeiro, INSTICC, Portugal 


\section{Program Committee}

Marco Aiello, University of Groningen, The Netherlands

Dietrich Albert, University of Graz \& Graz University of Technology, Austria

Carlo Alberto Avizzano, Scuola Superiore Sant' Anna, Italy

Angel Barriga, IMSE-CNM-CSIC, Spain

Karsten Berns, Robotics Research Lab, Germany

Frada Burstein, Faculty of IT, Monash University, Australia

Stuart Cunningham, Glyndwr University, U.K.

Mary Czerwinski, Microsoft Research, U.S.A.

Maria Luisa Damiani, Università degli Studi di Milano, Italy

Monica Divitini, Norwegian University of Science \& Technology, Norway

Deborah I. Fels, Ryerson University, Canada

Jinjuan Heidi Feng, Towson University (Baltimore Hebrew University), U.S.A.

Ana Fred, Instituto de Telecomunicações / IST, Portugal

Diamantino Freitas, Faculdade de Engenharia da Universidade do porto, Portugal

Ennio Gambi, Università Politecnica Delle Marche, Italy

Todor Ganchev, Technical University of Varna, Bulgaria

Jaakko Hakulinen, University of Tampere, Finland

Dirk Heylen, Human Media Interaction, University of Twente, The Netherlands

Alina Huldtgren, Eindhoven University of Technology, Germany

Kyoung-Don Kang, Binghamton University, U.S.A.

David Kaufman, Simon Fraser University, Canada

Takahiro Kawamura, Toshiba Corp., Japan
Peter Kokol, University of Maribor, Slovenia

Herman van der Kooij, University of Twente, The Netherlands

Andrej Kos, University of Ljubljana, Slovenia

Mikel Larrea, Universidad del País Vasco, Spain

Barbara Leporini, ISTI - CNR, Italy

Christine Lisetti, Florida International University, U.S.A.

Linda Little, Northumbria University, U.K.

Thurmon Lockhart, Arizona State University, U.S.A.

Piero Malcovati, University of Pavia, Italy

Roberto Manduchi, University of California Santa Cruz, U.S.A.

Kathleen F. McCoy, University of Delaware, U.S.A.

Nirvana Meratnia, University of Twente, The Netherlands

Hiroshi Mineno, Shizuoka University, Japan

Elena Mugellini, University of Applied Sciences Western Switzerland, Switzerland

Majid Nabi, Eindhoven University of Technology, The Netherlands

Keiichi Nakata, University of Reading, U.K.

Amit Anil Nanavati, IBM Research, India

Lorenzo Natale, Istituto Italiano di Tecnologia, Italy

Anthony F. Norcio, UMBC (University of Maryland Baltimore County), U.S.A.

Masayuki Numao, Osaka University, Japan

Angelo Oddi, Consiglio Nazionale delle Ricerche, Italy

John O'Donoghue, Imperial College London, U.K.

Patrizio Pelliccione, University of Gothenburg, Sweden

Ronald H. Perrott, Oxford e-Research Centre, U.K.

Niels Pinkwart, Humboldt University, Germany 


\section{Program Committee (CONT.)}

Ronald Poppe, Utrecht University, The Netherlands

Marco Porta, Università degli Studi di Pavia, Italy

Girijesh Prasad, University of Ulster, U.K.

Klaus Radermacher, RWTH Aachen University, Germany

Bernd Radig, Technische Universitaet Muenchen, Germany

Gregorio Robles, Universidad Rey Juan Carlos, Spain

Wendy Rogers, Georgia Institute of Technology, U.S.A.

Philippe Roose, LIUPPA/IUT de Bayonne/UPPA, France

Corina Sas, Lancaster University, U.K.

Sreela Sasi, Gannon University, U.S.A.

Fabio A. Schreiber, Politecnico di Milano, Italy

Loren Schwiebert, Wayne State University, U.S.A.
Bryan W. Scotney, University of Ulster, U.K.

Yuzhong Shen, Old Dominion University, U.S.A.

Josep Silva, Universidad Politécnica de Valencia, Spain

Luca Spalazzi, Università Politecnica Delle Marche, Italy

Christine Strauss, University of Vienna, Austria

Frans R. J. Verhey, Maastricht University, The Netherlands

Salvatore Vitabile, Università degli Studi di Palermo, Italy

Gerhard Weber, Technical University of Dresden, Germany

Martin White, University of Sussex, U.K.

George Xylomenos, Athens University of Economics and Business, Greece

Xenophon Zabulis, Foundation for Research and Technology Hellas (FORTH), Greece

\section{AuXiliary ReVieWER}

Lucia Pepa, DII, Dipartimento di Ingegneria dell'Informazione (UNIVPM), Italy 


\section{Special Sessions Program Committee}

\section{Special Session on How Can Digital Games Help Older Adults?}

Emmanuel Duplaa, University of Ottawa, Canada

Ana Fred, Instituto de Telecomunicações / IST, Portugal

John O'Donoghue, Imperial College London, U.K.

Lise Renaud, Universite de Quebec a Montreal, Canada
Carsten Röcker, Ostfestfalen-Lippe UAS and Fraunhofer IOSB-INA, Germany

Michelle Vanchu-Orosco, Simon Fraser University, Canada

Martina Ziefle, RWTH-Aachen University, Germany

\section{Special Session on Social Motivations and Social Benefits of ICT}

António Cunha, UTAD - Universidade de Trás-os-Montes e Alto Douro, Portugal

Emilia Duarte, IADE-U - Instituto de Arte, Design e Empresa - Universitário, Portugal

Bernardo Providência, Universidade do Minho, Portugal
Joao Rodrigues, University of the Algarve, Portugal

João Varajão, University of Trás-os-Montes e Alto Douro, Portugal

\section{Selected Papers Book}

A number of selected papers presented at ICT4AgeingWell 2015 will be published by Springer-Verlag in a CCIS Series book. This selection will be done by the Conference Co-chairs and Program Co-chairs, among the papers actually presented at the conference, based on a rigorous review by the ICT4AgeingWell 2015 Program Committee members. 


\section{FOREWORD}

This book contains the proceedings of the International Conference on Information and Communication Technologies for Ageing Well and e-Health (ICT4AgeingWell 2015) which was organized and sponsored by the Institute for Systems and Technologies of Information, Control and Communication (INSTICC) and held in cooperation with the International Society for Telemedicine \& eHealth - ISfTeH, European Health Telematics Association EHTEL and AAL Programme.

ICT4AgeingWell aims to be an annual meeting point for those that study and apply Information and Communication Technologies for improving the quality of life of the elderly and for helping people stay healthy, independent and active at work or in their community along their whole life. ICT4AgeingWell facilitates the exchange of information and dissemination of best practices, innovation and technical improvements in the fields of age-related health care, education, social coordination and ambient assisted living.

This conference brought together researchers and practitioners interested in methodologies and applications related to the Information and Communication Technologies for Ageing Well and e-Health fields. It had five main topic areas, covering different aspects, including Ambient Assisted Living, Telemedicine and E-Health, Monitoring, Accessibility and User Interfaces, Robotics and Devices for Independent Living and HCI for Ageing Populations. We believe these proceedings demonstrate new and innovative solutions, and highlight technical problems in each field that are challenging and worthwhile.

ICT4AgeingWell 2015 received 45 paper submissions from 28 countries in all continents, of which $27 \%$ were accepted as full papers. The high quality of the papers received imposed difficult choices in the review process. To evaluate each submission, a double blind paper review was performed by the Program Committee, whose members are highly qualified independent researchers in the ICT4AgeingWel 2015 topic areas.

The high quality of the ICT4AgeingWell 2015 programme was enhanced by four keynote lectures, delivered by experts in their fields, including (alphabetically): Juan Carlos Augusto (School of Science and Technology, Middlesex University, United Kingdom), Thomas Hermann (CITEC - Center of Excellence Cognitive Interaction Technology, Bielefeld University, Germany), Victor Chang (Leeds Beckett University, United Kingdom) and William Molloy (Centre for Gerontology and Rehabilitation, School of Medicine, UCC, Ireland).

The meeting was also complemented with a Doctoral Consortium on "Information and Communication Technologies for Ageing Well and e-Health" chaired by Ana Fred, Instituto de Telecomunicações / IST, Portugal.

All presented papers will be submitted for indexation by Thomson Reuters Conference Proceedings Citation Index (ISI), INSPEC, DBLP, EI (Elsevier Index) and Scopus, as well as being made available at the SCITEPRESS Digital Library. Furthermore, a short list of 
presented papers will be selected and their authors will be invited to submit an extended version of their papers to be included in a forthcoming book of ICT4AgeingWell Selected Papers to be published in CCIS Series by Springer Verlag during 2015.

The best contributions to the conference and the best student submissions were distinguished with awards based on the best combined marks of paper reviewing, as assessed by the Program Committee, and the quality of the presentation, as assessed by session chairs at the conference venue.

The conference was complemented with two special sessions, one focusing on Digital Games, named Special Session on How Can Digital Games Help Older Adults? - AGEWELL 2015, chaired by David Kaufman, Simon Fraser University, Canada and the other one focusing on Social Motivations and Social Benefits of ICT named Special Session on Social Motivations and Social Benefits of ICT - SocialICT 2015, chaired by Paula Trigueiros, University of Minho, Portugal.

Building an interesting and successful program for the conference required the dedicated effort of many people. We would like to express our thanks, first of all, to all authors including those whose papers were not included in the program. We would also like to express our gratitude to all members of the Program Committee and auxiliary reviewers, who helped us with their expertise and valuable time. Furthermore, we thank the invited speakers for their invaluable contribution and for taking the time to synthesize and prepare their talks.

Finally, we gratefully acknowledge the professional support of the INSTICC team for all organizational processes.

\title{
Markus Helfert
}

Dublin City University, Ireland

\author{
Andreas Holzinger \\ Medical University Graz, Austria
}

\section{Martina Ziefle}

RWTH-Aachen University, Germany

\section{Ana Fred}

Instituto de Telecomunicações / IST, Portugal

\section{John O'Donoghue}

Imperial College London, U.K.

\section{Carsten Röcker}

Ostfestfalen-Lippe UAS and Fraunhofer IOSB-INA, Germany 


\section{CONTENTS}

\section{INVITED SPEAKERS}

\section{KEYNOTE SPEAKERS}

Developing User-centric AAL Systems

Juan Carlos Augusto

Cloud Computing and Big Data Can Improve the Quality of Our Life

Victor Chang

Auditory Displays for Ambient Intelligence - Perspectives for Smart Environments

Thomas Hermann

Community Assessment of Risk Screening and Treatment Strategies (CARTS) - An Update

William Molloy

\section{AMBIENT ASSISTED LIVING}

\section{Full Papers}

Assistive Technology for Risks Affecting Elderly People in Outdoor Environment Hady Khaddaj Mallat, Rami Yared and Bessam Abdulrazak

Toward Pervasive Computing System to Enhance Safety of Ageing People in Smart Kitchen Bessam Abdulrazak, Rami Yared, Thomas Tessier and Philippe Mabilleau

\section{ShORT PAPERS}

Requirements for Relaxation Coaching - A Formalization of the Fogg Behavior Model Rogier M. van Eijk

Personalization of Virtual Coaching Applications using Procedural Modeling

René Zmugg, Andreas Braun, Peter Roelofsma, Wolfgang Thaller, Lisette Moeskops, Sven Havemann, Gabrijela Reljic and Dieter W. Fellner

An Integration Platform for Private Assisted Houses

Flavio Corradini, Francesco De Angelis, Barbara Re, Emiliano Anceschi, Massimo Callisto De Donato and Paolo Iddas

Ambient Assisted Living Buddy

Alexiei Dingli and Michael Buhagiar

Augmented Hearing Assistance for Elderly People - From Requirements to Implementation

Pierre Barralon, Ivo Ramos Maia Martins, Nicole Merkle and Stephanie Schwarz

Towards the Deployment of Open Platform AAL Services in Real Life-advantages and Lessons Learned - uSmAAL: A Case Study for Implementing Intelligent AAL Services in Real Life based on the Open Platform universAAL

Jan Stengler, Gouri Gaikward and Helmi Ben Hmida

Ageing and Death - Breaking a Taboo

Matthias Rauterberg and Kiarash Irandoust 
Continuous Monitoring and Digital Systems for Elders

Nuno Rocha, Raquel Sousa and Gil Gonçalves

\section{TELEMEDICINE AND E-HEALTH}

\section{Full Papers}

Telegeriatrics - A Pilot Project to Reduce Unnecessary Hospital Admissions of Nursing Home Residents in Singapore

H. J. Toh, Chia J., E. Koh, K. Lam, G. C. Magpantay, C. M. De Leon and J. A. Low

User Perceptions of the Telemedicine Programme in Nursing Homes - The Singapore Perspective H. J. Toh, J. Chia, E. Koh, K. Lam, G. C. Magpantay, C. M. De Leon and J. A. Low

On Modeling the Cardiovascular System and Predicting the Human Heart Rate under Strain Melanie Ludwig, Ashok Meenakshi Sundaram, Matthias Füller, Alexander Asteroth and Erwin Prassler

Towards Enhancing Communication Between Caregiver Teams and Elderly Patients in Emergency Situations

Syed Atif Mehdi, Artem Avtandilov, Shah Rukh Humayoun and Karsten Berns

\section{Short PAPERS}

BigTexts - A Framework for the Analysis of Electronic Health Record Narrative Texts based on Big Data Technologies

Wilson Alzate Calderón, Alexandra Pomares Quimbaya, Rafael A. Gonzalez and Oscar Mauricio Muñoz

Reliability and Usability of the eChez-Soi In-home Telerehabilitation Platform: A New Internet-based Communication and Real-time Monitoring Software Solution Combined with Interactive Exercises - Results of a Longitudinal Pilot Study in Four Patients with Lung Cancer Hélène Moffet, Didier Saey, Valérie Coats, Claude Vincent, Fanny Choinière and François Comeau

NHIS Big Data and Health Services - Consolidated Ageing Well Strategy in Korea Soon-Ae Shin, Dong-Soo Choo, Tae-Hwa Han, Jong-Heon Park, Jong-Hee Kim and Ji-Hyon Bang

Multimodal Analysis for Behavioural Recognition in Tele-assistance Applications

Sorin Soviany and Sorin Puscoci

\section{MONITORING, ACCESSIBILITY AND USER INTERFACES}

\section{Full Paper}

Improving Activity Monitoring Through a Hierarchical Approach

Xavier Rafael-Palou, Eloisa Vargiu, Guillem Serra and Felip Miralles

\section{Short PAPers}

User-sentiment based Evaluation for Market Fitness Trackers - Evaluation of Fitbit One, Jawbone Up and Nike+ Fuelband based on Amazon.com Customer Reviews

Hassan Issa, Alaa Shafaee, Stefan Agne, Stephan Baumann and Andreas Dengel

A Position Paper Managing Youth Screen Time versus Physical Activity - Encouraging Active Living in a Technological Focussed Generation

Reem Altamimi, Keith Nesbitt and Geoff Skinner 
EPIK - Virtual Rehabilitation Platform Devised to Increase Self-reliance of People with Limited Mobility

Sonia Garrote, Azael J. Herrero, Miguel Pedraza-Hueso, Carlos González-Gutiérrez, María V. Fernández-San Román, F. J. Díaz-Pernas, Héctor Menéndez, Cristina M. Ferrero and Mario Martínez-Zarzuela

Perception of Human Activities - A Means to Support Connectedness Between the Elderly and Their Caregivers

Kadian Davis, Evans Owusu, Carlo Regazzoni, Lucio Marcenaro, Loe Feijs and Jun Hu

Unobtrusive Monitoring of Physical Activity in AAL - A Simple Wearable Device Designed for Older Adults

Adelmo De Santis, Antonio Del Campo, Ennio Gambi, Laura Montanini, Giovanni Pelliccioni, Davide Perla and Susanna Spinsante

\section{ROBOTICS AND DEVICES FOR INDEPENDENT LIVING}

\section{ShORT PAPERS}

Scenario Development for Successful Aeging with Robot Companions Katja Richter and Nicola Döring

A TV-based ICT Platform for Active Ageing, Tele-care and Social Networking

Silvia Macis, Daniela Loi, Danilo Pani, Wil Rijnen and Luigi Raffo

\section{HCI FOR AGEING POPULATIONS}

\section{Full Papers}

Reminiscence Map - Insights to Design for People with Dementia from a Tangible Prototype Alina Huldtgren, Anja Vormann and Christian Geiger

Interaction Modeling in PRACTICE - CTT Vs. SCXML - A Comparison of Two Practical Solutions Applying Interaction Modeling Techniques for Multimodal User-System Interaction

Miroslav Sili, Matthias Gira, Markus Müllner-Rieder and Christopher Mayer

\section{ShORT PAPERS}

The Assistant Project - Creating a Smartphone App to Assist Older People when Travelling by Public Transport

Philip Barham, Stefan Carmien and Ainara Garzo

ICT Solutions to Develop an Effective Motor and Cognitive Training to Reduce Risk of Falls The I-DONT-FALL Project

Francesco Barban, Roberta Annicchiarico, Alessia Federici, Ilenia Debora Mazzù, Maria Giovanna Lombardi, Simone Giuli, Claudia Ricci, Fulvia Adriano, Ivo Griffini, Manuel Silvestri, Massimo Chiusso, Sergio Neglia, Raquel Cuevas Perez, Yannis Dionyssiotis, Georgios Koumanakos, Milo Kovačeić, Nuria Montero, Oscar Pino, Carmela Zincarelli, Niels Boye, Cristian Barrué, Peter Levene, Stelios Pantelopoulos, Roberto Rosso, Angelo Maria Sabatini and Carlo Caltagirone 


\section{SPECIAL SESSION ON HOW CAN DIGITAL GAMES HELP OLDER ADULTS?}

\section{Full Papers}

Barriers and Adaptations of a Digital Game for Older Adults

Robyn Schell, Simone Hausknecht and David Kaufman

Building Seniors' Social Connections and Reducing Loneliness Through a Digital Game

Simone Hausknecht, Robyn Schell, Fan Zhang and David Kaufman

Computer Games for Older Adults beyond Entertainment and Training: Possible Tools for Early Warnings - Concept and Proof of Concept

Béla Pataki, Péter Hanák and Gábor Csukly

\section{ShORT PAPER}

Computer-assisted Intervention using Touch-screen Video Game Technology on Cognitive Function and Behavioural Symptoms for Community-dwelling Older Chinese Adults with Mild-to-Moderate Dementia - Preliminary Results of a Randomized Controlled Trial

Ruby Yu, Dawn Poon, Ashley Ng, Kitty Sit, Jenny Lee, Bosco Ma, CM Lum, Fannie Yeung, Martin Wong, Elsie Hui and Jean Woo

\section{SPECIAL SESSION ON SOCIAL MOTIVATIONS AND SOCIAL BENEFITS OF ICT}

\section{Short PAPers}

A Mobile Guardian Angel Supporting Urban Mobility for People with Dementia - An Errorless Learning based Approach

Laura Freina and Ilaria Caponetto

Eudaimonia in Human Factors Research and Practice - Foundations and Conceptual Framework Applied to Older Adult Populations

Katie Seaborn, Peter Pennefather and Deborah I. Fels

MindGym Strategies for Elderly People

Marjan Gusev, Sasko Ristov, Jurij Tasic, Darja Rudan Tasic, Shushma Patel and Dilip Patel

Exergames for Assessment in Active and Healthy Aging - Emerging Trends and Potentialities Evdokimos I. Konstantinidis, Panagiotis E. Antoniou and Panagiotis D. Bamidis 